24 December 2001

OU $-397 / 2001$

NUC-MINN-01/17-T

\title{
Cosmic Shells
}

\author{
Yutaka Hosotani and Takayuki Nakajima \\ Department of Physics, Osaka University, Toyonaka, Osaka 560-0043, Japan
}

\author{
Ramin G. Daghigh and Joseph I. Kapusta \\ School of Physics and Astronomy, University of Minnesota, Minneapolis, MN 55455, USA
}

\begin{abstract}
When a potential for a scalar field has two local minima there arise spherical shell-type solutions of the classical field equations due to gravitational attraction. We establish such solutions numerically in a space which is asymptotically de Sitter. It generically arises when the energy scale characterizing the scalar field potential is much less than the Planck scale. It is shown that the mirror image of the shell appears in the other half of the Penrose diagram. The configuration is smooth everywhere with no physical singularity.
\end{abstract}




\section{Introduction}

Gravitational interactions, which are inherently attractive for ordinary matter, can produce soliton-like objects even when such things are strictly forbidden in flat space. They are possible as a consequence of the balance between repulsive and attractive forces. One such example is a monopole or dyon solution in the pure Einstein-Yang-Mills theory in the asymptotically anti-de Sitter space [1, 2, 3]. In the pure Yang-Mills theory in flat space there can be no static solution at all [4] but once gravitational interaction is included there arise particle-like solutions [5]. Whereas all solutions are unstable in the asymptotically flat or de Sitter space, there appear a continuum of stable monopole and dyon solutions in the asymptotically anti-de Sitter space. The stable solutions are cosmological in nature; their size is typically of order $|\Lambda|^{-1 / 2}$ where $\Lambda$ is the cosmological constant.

The possibility of false vacuum black holes has also been explored. Suppose that the potential in a scalar field theory has two minima, one corresponding to the true vacuum and the other to the false vacuum. If the universe is in the false vacuum, a bubble of the true vacuum is created by quantum tunneling which expands with accelerated velocity. The configuration is called a bounce [6]. Now flip the configuration [7]. The universe is in the true vacuum with potential $V=0$ and the inside of a sphere is excited to the false vacuum with $V>0$. Is such a de Sitter lump in Minkowski space possible? If the lump is too small it would be totally unstable. The energy localized inside the lump can dissipate to spatial infinity. If the lump is big enough the Schwarzschild radius becomes larger than the lump radius so that the lump is inside a black hole. The energy cannot escape to infinity. It looks like a soliton in Minkowski space. However, as a black hole it is a dynamical object. The configuration is essentially time-dependent. This false vacuum black hole configuration, however, does not solve the equations at the horizon. It has been recently proven that there can be no such everywhere-regular black hole solution [8, 9]. Rather, false vacuum lumps in flat space evolve dynamically [10, 11, 12].

The purpose of this paper is to report new solutions to the coupled equations of gravity and scalar field theory which display spherically symmetric shell structure [13]. We demonstrate that such structure appears when the potential for a scalar field has two local minima and the space is asymptotically de Sitter. In the examples we present, both the inside and outside of the shells are de Sitter space with the same cosmological constant. 
The structure becomes possible only when the energy scale of the scalar field potential becomes small compared with the Planck scale. While such shell structures might not be easy to create in the present universe, it is quite plausible that they could have been created during a phase transition early in the universe [10]. A similar configuration has been investigated in ref. [14].

The plan of our paper is as follows. In section 2 we precisely state the problem, solve the field equations in those regions of space-time where they can be linearized, and sketch the solution in the nonlinear shell region in static coordinates. In section 3 we solve the nonlinear equations in the shell region and display the dependence on the parameters of the theory. In section 4 we extend the solution from static coordinates, which have a coordinate singularity, to other coordinate systems that do not, thereby displaying the existence and character of the solution throughout the full space-time manifold. In section 5 we study the stability of the classical solution to quantum fluctuations. Our summary and conclusions are given in section 6 .

\section{Shell Solutions in Static Coordinates}

Consider a scalar field coupled to gravity with the Lagrangian

$$
\mathcal{L}=\frac{1}{16 \pi G} R+\frac{1}{2} \phi_{; \mu} \phi^{; \mu}-V[\phi]
$$

where the potential $V[\phi]$ has two minima at $f_{1}$ and $f_{2}$ separated by a barrier. See fig. 1 .

We look for spherically symmetric configurations in which the metric of space-time is written as

$$
d s^{2}=-\frac{H}{p^{2}} d t^{2}+\frac{d r^{2}}{H}+r^{2}\left(d \theta^{2}+\sin ^{2} \theta d \varphi^{2}\right)
$$

The functions $\phi, H$, and $p$ depend only on $r$ and $t$. A tetrad basis is chosen, in a region $H>0$, as

$$
e_{0}=\frac{\sqrt{H}}{p} d t, \quad e_{1}=\frac{1}{\sqrt{H}} d r, \quad e_{2}=r d \theta, \quad e_{3}=r \sin \theta d \varphi .
$$

The components of the energy-momentum tensor in the tetrad basis, $T_{a b}=e_{a}{ }^{\mu} e_{b}{ }^{\nu} T_{\mu \nu}$, are

$$
\begin{aligned}
& T_{00}=\frac{1}{2}\left(\frac{p^{2}}{H} \dot{\phi}^{2}+H \phi^{\prime 2}\right)+V[\phi] \\
& T_{11}=\frac{1}{2}\left(\frac{p^{2}}{H} \dot{\phi}^{2}+H \phi^{\prime 2}\right)-V[\phi]
\end{aligned}
$$




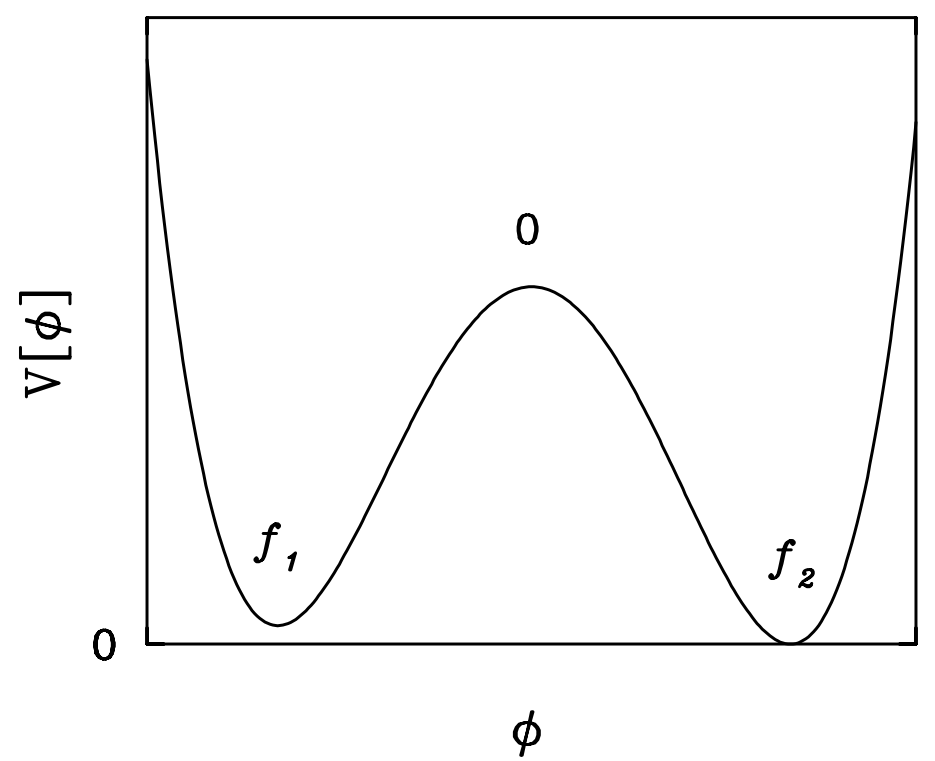

Figure 1: The potential $V[\phi]$.

$$
\begin{aligned}
& T_{22}=T_{33}=\frac{1}{2}\left(\frac{p^{2}}{H} \dot{\phi}^{2}-H \phi^{\prime 2}\right)-V[\phi] \\
& T_{01}=-p \dot{\phi} \phi^{\prime} .
\end{aligned}
$$

Here dot and prime indicate $t$ - and $r$-derivatives, respectively.

The scalar field satisfies

$$
p \frac{\partial}{\partial t}\left(\frac{p}{H} \dot{\phi}\right)-\frac{p}{r^{2}} \frac{\partial}{\partial r}\left(\frac{r^{2} H}{p} \phi^{\prime}\right)+V^{\prime}[\phi]=0 .
$$

We introduce the integrated mass function $M(t, r)$ by

$$
H=1-\frac{2 G M}{r}
$$

The Einstein equations are

$$
\begin{aligned}
& M=\int_{0}^{r} 4 \pi r^{2} d r T_{00}, \\
& \frac{p^{\prime}}{p}=-4 \pi G r\left\{\frac{p^{2}}{H^{2}} \dot{\phi}^{2}+\phi^{\prime 2}\right\}, \\
& \frac{\dot{H}}{H}=-8 \pi G r \dot{\phi} \phi^{\prime}, \\
& \frac{p}{2}\left\{\frac{\partial}{\partial t}\left(\frac{p \dot{H}}{H^{2}}\right)+\frac{\partial}{\partial r}\left(\frac{H^{\prime}}{p}-\frac{2 H p^{\prime}}{p^{2}}\right)\right\}+\frac{1-H}{r^{2}}=4 \pi G\left(\frac{p^{2}}{H} \dot{\phi}^{2}-H \phi^{\prime 2}\right) .
\end{aligned}
$$


One of the equations, Eq. (2.10), is redundant as it follows from Eqs. (2.5), (2.7), (2.8), and (2.9).

We shall seek static solutions for which the set of equations reduces to

$$
\begin{aligned}
& \phi^{\prime \prime}(r)+\Gamma_{\mathrm{eff}}(r) \phi^{\prime}(r)=\frac{1}{H} V^{\prime}[\phi], \\
& M(r)=\int_{0}^{r} 4 \pi r^{2} d r\left\{\frac{1}{2} H \phi^{2}+V[\phi]\right\},
\end{aligned}
$$

where

$$
\Gamma_{\mathrm{eff}} \equiv \frac{2}{r}+4 \pi G r \phi^{\prime 2}+\frac{H^{\prime}}{H}
$$

Eq. (2.11) can be interpreted as an equation for a particle with a coordinate $\phi$ and time $r$. Except for a factor $1 / H$ this particle moves in a potential $U[\phi]=-V[\phi]$. The coefficient $\Gamma_{\text {eff }}(r)$ represents time $(r)$-dependent friction.

The potential $V[\phi]$ is supposed to have two minima, at $f_{1}$ and $f_{2}$. We are looking for a solution which starts at $\phi \sim f_{1}$, moves close to $f_{2}$, and comes back to $f_{1}$ at $r=\infty$. The particle's potential $U[\phi]$ has two maxima. The particle begins near the top of one hill, rolls down into the valley and up the other hill, turns around and rolls down and then back up to the top of the original hill. This is impossible in flat space, as $\Gamma_{\text {eff }}$ is positive-definite so that the particle's energy dissipates and it cannot climb back to its starting point.

In the presence of gravity the situation changes. The non-vanishing energy density can make $H$ a decreasing function of $r$ so that $\Gamma_{\text {eff }}$ becomes negative. The energy lost by the particle during the initial rolling down can be regained on the return path by negative friction, or thrust. Indeed, this happens.

Let us set up the problem more precisely. We take a quartic potential with $f_{1}<0<f_{2}$;

$$
\begin{aligned}
V^{\prime}[\phi] & =\lambda \phi\left(\phi-f_{1}\right)\left(\phi-f_{2}\right) \\
V[\phi] & =\frac{\lambda}{4}\left(\phi-f_{2}\right)\left\{\phi^{3}-\frac{1}{3}\left(f_{2}+4 f_{1}\right) \phi^{2}-\frac{1}{3} f_{2}\left(f_{2}-2 f_{1}\right)\left(\phi+f_{2}\right)\right\} .
\end{aligned}
$$

Here $V\left[f_{2}\right]=0$, and $V[0]$ is a local maximum for the barrier separating the two minima. Define $f=\left(\left|f_{1}\right|+f_{2}\right) / 2$ and $\Delta f=f_{2}-\left|f_{1}\right|$. In case $\Delta f>0, \phi=f_{1}$ corresponds to a false vacuum with the energy density $\epsilon=V\left[f_{1}\right]=\frac{2}{3} \lambda f^{3} \Delta f>0$, whereas $\phi=f_{2}$ corresponds to a true vacuum with a vanishing energy density. As we shall discuss in detail below, the positivity of the energy density $\epsilon$ plays an important role for the presence of shell structure, 
but the vanishing $V\left[f_{2}\right]$ is not essential as we see below. In a more general potential it could be that $V\left[f_{2}\right]>V\left[f_{1}\right]$.

We look for solutions with $\phi$ starting at the origin $r=0$ very close to $f_{1}$. There is only one parameter to adjust: $\phi_{0} \equiv \phi(0)$. The behavior of a solution near the origin is given by

$$
\begin{array}{ll}
\phi=\phi_{0}+\phi_{2} r^{2}+\cdots, & \phi_{2}=\frac{1}{6} V^{\prime}\left[\phi_{0}\right], \\
p=1+p_{4} r^{4}+\cdots, & p_{4}=-4 \pi G \phi_{2}^{2}, \\
M=m_{3} r^{3}+\cdots, & m_{3}=\frac{4 \pi}{3} V\left[\phi_{0}\right], \\
H=1-2 G m_{3} r^{2}+\cdots . &
\end{array}
$$

Given $\phi_{0}$ the equations determine the behavior of a configuration uniquely. For most values of $\phi_{0}$ the corresponding configurations are unacceptable. As $r$ increases, $\phi(r)$ either approaches 0 (the local maximum of $V[\phi]$ ) or goes to $-\infty$. Other than the two trivial solutions, corresponding to the false and true vacua, we have found a new type of solution.

There are four parameters in the model, one of which, the gravitational constant $G=m_{\mathrm{P}}^{-2}$, sets the scale. The other three are $\lambda, f_{1}$, and $f_{2}$ or, equivalently, the three dimensionless quantities $f / m_{\mathrm{P}}, \Delta f / f$, and $\lambda$. We have explored only a limited region in the parameter space. The moduli space of solutions depends critically on $f / m_{\mathrm{P}}$ and $\Delta f / f$, but seems to depend little on $\lambda$. Nontrivial solutions appear as $f / m_{\mathrm{P}}$ becomes small.

If $\phi_{0}<f_{1}, \phi(r)$ monotonically decreases as the radius increases to diverge to $-\infty$. On the way $H(r)$ crosses zero. Suppose instead that $f_{1}<\phi_{0}<0$ and $\phi_{0}$ is not too close to $f_{1}$. In the particle analogue, the particle starts to roll down the hill under the action of $U[\phi]=-V[\phi]$. It approaches $\phi=0$, and oscillates around it. In the meantime $H(r)$ crosses zero.

Now suppose that $\phi_{0}$ is very close to, but still greater than, $f_{1}: \phi(0)=f_{1}+\delta \phi(0)$ with $0<\delta \phi(0) / f \ll 1$. A schematic of the resulting solution is displayed in fig. 2 and fig. 3 . We divide space into three regions in the static coordinates: region $\mathrm{I}\left(0 \leq r<R_{1}\right)$, region II $\left(R_{1}<r<R_{2}\right)$, and region III $\left(R_{2}<r\right)$. It turns out that $\phi(r)$ varies little from $f_{1}$ in regions I and III so that the equation of motion for $\phi$ may be linearized in those regions. In region II the field deviates strongly and the full set of nonlinear equations must be solved numerically. This is the region in which we shall find shell structure. $H(r)$ deviates from the de Sitter value significantly. In region III the spacetime is approximately de Sitter again. $H(r)$ crosses zero at $r_{H}$. 


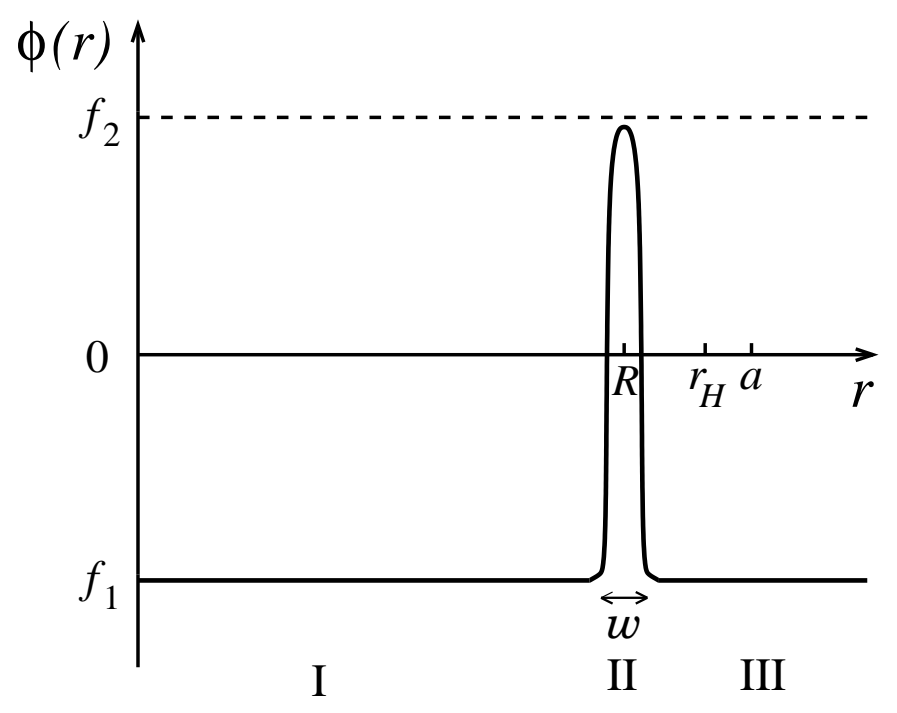

Figure 2: Schematic behavior of $\phi(r)$.

In region I the space-time is approximately de Sitter.

$$
T_{00}=\epsilon, \quad H=1-\frac{r^{2}}{a^{2}}, \quad a=\sqrt{\frac{3}{8 \pi G \epsilon}}, \quad p=1 .
$$

The equation for $\phi(r)$ can be linearized with $\phi(r)=f_{1}+\delta \phi(r)$. In terms of $z \equiv r^{2} / a^{2}$,

$$
\left\{z(1-z) \frac{d^{2}}{d z^{2}}+\left(\frac{3}{2}-\frac{5}{2} z\right) \frac{d}{d z}-\frac{1}{4} \omega^{2} a^{2}\right\} \delta \phi=0,
$$

where $\omega^{2}=V^{\prime \prime}\left[f_{1}\right]$. This is Gauss' hypergeometric equation. The solution which is regular at $r=0$ is

$$
\delta \phi(r)=\delta \phi(0) \cdot F\left(\frac{3}{4}+i \kappa, \frac{3}{4}-i \kappa, \frac{3}{2} ; z\right)
$$

where

$$
\kappa=\frac{1}{2} \sqrt{\omega^{2} a^{2}-\frac{9}{4}}, \quad \omega^{2} a^{2}=\frac{9 m_{\mathrm{P}}^{2}}{8 \pi f \Delta f}\left(1-\frac{\Delta f}{2 f}\right) .
$$

We shall soon see that a solution with shell structure appears for $\omega a \gg 1$ with a particular choice of $\delta \phi(0)$. The ratio of $\delta \phi^{\prime}(r)$ to $\delta \phi(r)$ is given by

$$
\frac{\delta \phi^{\prime}(r)}{\delta \phi(r)}=\frac{4 r}{3 a^{2}}\left(\kappa^{2}+\frac{9}{16}\right) \frac{F\left(\frac{7}{4}+i \kappa, \frac{7}{4}-i \kappa, \frac{5}{2} ; z\right)}{F\left(\frac{3}{4}+i \kappa, \frac{3}{4}-i \kappa, \frac{3}{2} ; z\right)} \equiv \frac{2 r}{a^{2}} J(z) .
$$




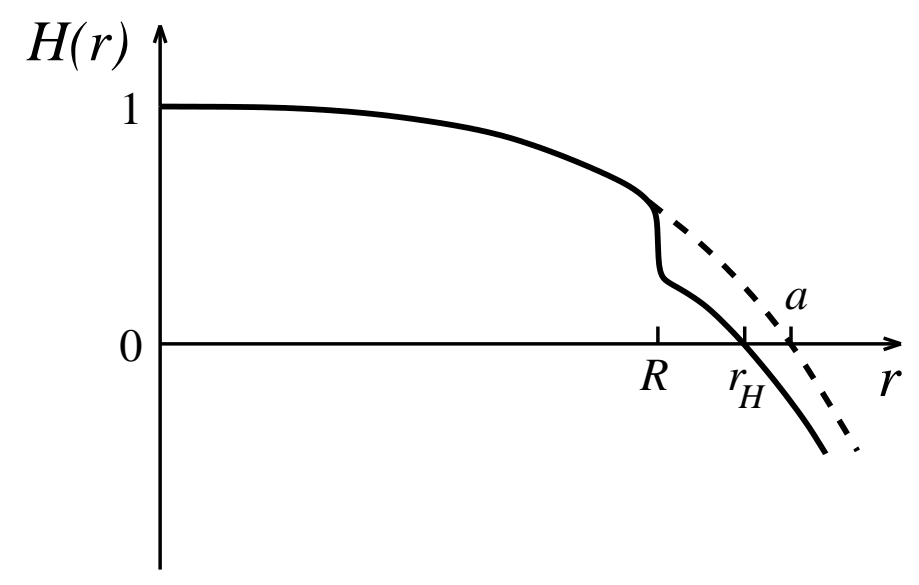

Figure 3: Schematic behavior of $H(r)$.

The deviation from $f_{1}$ at the origin, $\delta \phi(0)$, needs to be very small for an acceptable solution. The behavior of the hypergeometric function for $\kappa \gg 1$ and $0<z<1$ is given by 15

$$
F(a+i \kappa, a-i \kappa, c ; z) \sim \frac{\Gamma(c)}{2 \sqrt{\pi}} \kappa^{\frac{1}{2}-c} z^{-\frac{c}{2}+\frac{1}{4}}(1-z)^{\frac{c}{2}-\frac{1}{4}-a} \exp \left\{2 \kappa \sin ^{-1} \sqrt{z}\right\} .
$$

The ratio $\delta \phi(r) / \delta \phi(0)$ grows exponentially as $r$ increases like $(4 \kappa)^{-1} z^{-1 / 2}(1-$ $z)^{-1 / 4} \exp \left\{2 \kappa \sin ^{-1} \sqrt{z}\right\}$. At the end of region I, $\delta \phi /\left|f_{1}\right|$ needs to be very small for the linearization to be valid. The ratio of $F^{\prime}(z)$ to $F(z), J(z)$ in (2.20), is given by

$$
J(z)=\frac{\kappa}{\sqrt{z(1-z)}} \quad \text { for } \kappa \gg 1,0<z<1 .
$$

In region II, $\phi(r)$ varies substantially and the nonlinearity of the equations plays an essential role. In this region the equations must be solved numerically. With fine tuning of the value of $\delta \phi\left(R_{1}\right)$ nontrivial shell solutions will be found.

The algorithm is the following. First $\delta \phi\left(R_{1}\right)$ is chosen and $\delta \phi^{\prime}\left(R_{1}\right)$ is evaluated by (2.20) and (2.22). To the order in which we work the metric is $H\left(R_{1}\right)=1-\left(R_{1} / a\right)^{2}$ and $p\left(R_{1}\right)=1$. With these boundary conditions Eqs. (2.11) and (2.12) are numerically solved.

The behavior of solutions in region II is displayed in fig. 4 . When the specific values of the input parameters are chosen to be $\lambda=0.01, f / m_{\mathrm{P}}=0.002$, and $\Delta f / f=0.002$, then 
the output parameters are: $a / l_{\mathrm{P}}=2.365 \times 10^{7}, \kappa=3.344 \times 10^{3}, R_{1} / l_{\mathrm{P}}=1.76104695 \times 10^{7}$ and $\delta \phi\left(R_{1}\right) / m_{\mathrm{P}}=10^{-5}$. (Here $l_{\mathrm{P}}$ is the Planck length.) The field $\phi(r)$ approaches $f_{1}$ for $r>R_{2}$. In the numerical integration $\delta \phi\left(R_{1}\right)$ is kept fixed while $R_{1}$ is varied. Fine-tuning to the ninth digit is necessary! If $R_{1}$ is taken to be slightly bigger then $\phi(r)$ starts to deviate from $f_{1}$ in the negative direction, diverging to $-\infty$ as $r$ increases. If $R_{1}$ is taken to be slightly smaller then $\phi(r)$ starts to deviate from $f_{1}$ in the positive direction, heading for $f_{2}$ as $r$ increases. With just the right value the space-time becomes nearly de Sitter outside the shell. The value of $\delta \phi$ at the origin $(r=0)$ is found from 2.18 ) to be $1.40 \times 10^{-2440}$, which explains why one cannot numerically integrate $\phi(r)$ starting from $r=0$.

The behavior of the solution in region III is easily inferred. From the numerical integration in region II both $H_{2}=H\left(R_{2}\right)$ and $p_{2}=p\left(R_{2}\right)$ are determined. In region III the metric can be written in the form

$$
\begin{aligned}
& H(r)=1-\frac{2 G \tilde{M}}{r}-\frac{r^{2}}{a^{2}} \\
& p(r)=p_{2} .
\end{aligned}
$$

Here $\tilde{M}$ is the mass ascribable to the shell. Once $T_{00}$ vanishes $H(r)$ must take this form. The value of $\tilde{M}$ may be determined numerically by fitting Eq. (2.23) just outside the shell. The location of the horizon, $r_{H}$, is determined by $H\left(r_{H}\right)=0$. The field $\phi(r)$ remains very close to $f_{1}$ in region III.

At this point due caution is required to continue the solution because the static coordinates defined in (2.2) do not cover the whole of space-time. The global structure of the space-time and of the solutions is worked out in Section 4 where different coordinates are employed.

The solutions we found have shell structure at a radius $R$ whose width $w$ is very small. The radius $R$ is smaller than the horizon radius $r_{H}$, but is of the same order as $a$. In the next section we shall see that $R$ becomes smaller as $f$ becomes smaller, but remains of order $a$ even in the $f \rightarrow 0$ limit. This implies that the shell structure is cosmic in size.

An estimate of the order of magnitude of $w$ as well as the conditions necessary for the existence of the cosmic shell are obtained by a simple argument. Return to Eq. (2.11).

$$
H \phi^{\prime \prime}+\left(\frac{2 H}{r}+H^{\prime}\right) \phi^{\prime}+4 \pi G r H \phi^{\prime 3}=V^{\prime}[\phi]
$$


In the shell region (region II, $r \sim R$ ) of typical solutions, $H$ is of order one and drops sharply. In (2.24) the $2 H / r$ term is negligible compared with the $H^{\prime}$ term. The remaining terms, $H \phi^{\prime \prime}, H^{\prime} \phi^{\prime}$, and $V^{\prime}[\phi]$ are all of the same order of magnitude so that $f / w^{2} \sim \lambda f^{3}$ or

$$
w \sim \frac{1}{\sqrt{\lambda} f}
$$

Hence the thickness of the shell is determined by the parameters in the scalar field potential. The radius $R$ is smaller than but of the same order as $a$. The solutions exist only if $H^{\prime}$, which is negative, dominates over $4 \pi G r H \phi^{\prime 2}$. In other words $w^{-1}>4 \pi G R(f / w)^{2}$. Making use of $R \sim a, a^{2}=9 /\left(16 \pi G \lambda f^{3} \Delta f\right)$ and (2.25), one finds

$$
\begin{aligned}
& 4 \pi\left(\frac{f}{m_{\mathrm{P}}}\right)^{2}<\frac{w}{a} \sim \frac{4 \sqrt{\pi}}{3} \frac{\sqrt{f \Delta f}}{m_{\mathrm{P}}} \ll 1 \\
& \frac{\Delta f}{f}>3 \sqrt{\pi}\left(\frac{f}{m_{\mathrm{P}}}\right)^{2} .
\end{aligned}
$$

We shall see in the next section that these relations are satisfied in the solutions obtained numerically.

\section{Numerical Analysis of the Nonlinear Regime}

In the last section we solved the linearized field equation for $\phi(r)$ in regions I and III where the deviation from $f_{1}$ is small, and we sketched the behavior in the nonlinear region II where the shell structure appears. In this section we present numerical results for region II. As discussed in the last section the boundary between regions I and II, located at the matching radius $R_{1}$, is rather arbitrary, subject only to the condition that the linearization is accurate up to that radius. Precise tuning is necessary for the pair $R_{1}$ and $\delta \phi\left(R_{1}\right)$ to obtain a solution to all the equations. Technically it is easier to keep $\delta \phi\left(R_{1}\right)$ fixed and adjust the matching radius $R_{1}$. If $R_{1}$ is chosen too small $\phi$ comes back toward, but cannot reach, $f_{1}$. What happens is that $\phi$ eventually oscillates around $\phi=0$ as $r$ increases. If $R_{1}$ is chosen too large $\phi$ comes back to $f_{1}$ at finite $r$ to roll over it and decreases indefinitely toward $-\infty$. For each sufficiently small value of $\delta \phi$ chosen as the matching value there exists a desired solution with $R_{1}=R_{c}$. The critical value $R_{c}$ can be determined numerically to arbitrary accuracy. 


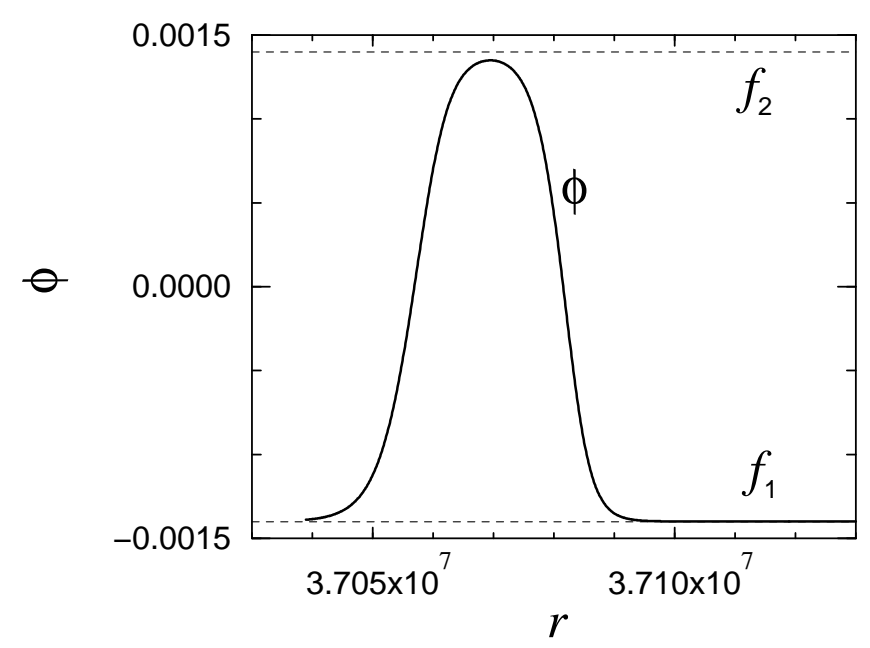

Figure 4: $\phi(r)$ of a solution with $f / m_{\mathrm{P}}=1.40 \times 10^{-3}, \Delta f / f=0.002, \lambda=0.01 . \quad \phi$ and $r$ are in the units of $m_{\mathrm{P}}$ and $l_{\mathrm{P}}$, respectively. The maximum value of $\phi$ is smaller than $f_{2} \sim 0.0014 \cdot m_{\mathrm{P}}$

One example of such a solution is displayed in fig. 1 for the parameters $\mathrm{f} / \mathrm{m}_{\mathrm{P}}=1.40 \times$ $10^{-3}, \Delta f / f=0.002, \lambda=0.01$ and $\delta \phi\left(R_{1}\right)=1.0 \times 10^{-5}$. The matching radius $R_{1}$ is fine-tuned to ten digits: $R_{1} / l_{\mathrm{P}} \sim 3.7038855228 \times 10^{7}$. The shell is very thin compared to the radius of the shell, lying in the region $3.704 \times 10^{7} \leq r / l_{\mathrm{P}} \leq 3.710 \times 10^{7}$.

In the shell region both $H(r)$ and $p(r)$ decrease in a two-step fashion. See figs. 5 and 6. Inside and outside the shell $H(r)$ is given by (2.16) and (2.23), whereas $p(r)$ assumes the constant values 1 and 0.6256 , respectively.

The change is induced by the non-vanishing energy-momentum tensor components $T_{00}=-T_{22}=-T_{33}$ and $T_{11}$. They are displayed in fig. 7 and fig. 8. The energy density $T_{00}$ has two sharp peaks associated with the rapid variation of $\phi$. The radial pressure $T_{11}$, on the other hand, steps up quickly, remains constant within the shell, and then steps down again. The value of $T_{11}$ is very small $\left(\sim 5 \times 10^{-17} m_{\mathrm{P}}^{4}\right)$ compared with the maximum value of $T_{00}\left(\sim 2 \times 10^{-14} m_{\mathrm{P}}^{4}\right)$. The contributions of the kinetic energy, $\frac{1}{2} H \phi^{\prime 2}$, and potential energy, $V[\phi]$, almost cancel each other.

In this example $\phi(r)$ makes a bounce transition $f_{1} \rightarrow f_{2} \rightarrow f_{1}$ once. As the numerical value of $f$ becomes smaller than $0.002 m_{\mathrm{P}}$ a new type of solutions with double bounces $f_{1} \rightarrow f_{2} \rightarrow f_{1} \rightarrow f_{2} \rightarrow f_{1}$ emerge. One example is displayed in fig. 9 .

The spectrum of the shell solutions depends on the parameter $f$. In fig. $10 R / a$, $r_{H} / a$, and $R / r_{H}$ are plotted as functions of $f$. (Finding the solution numerically becomes 


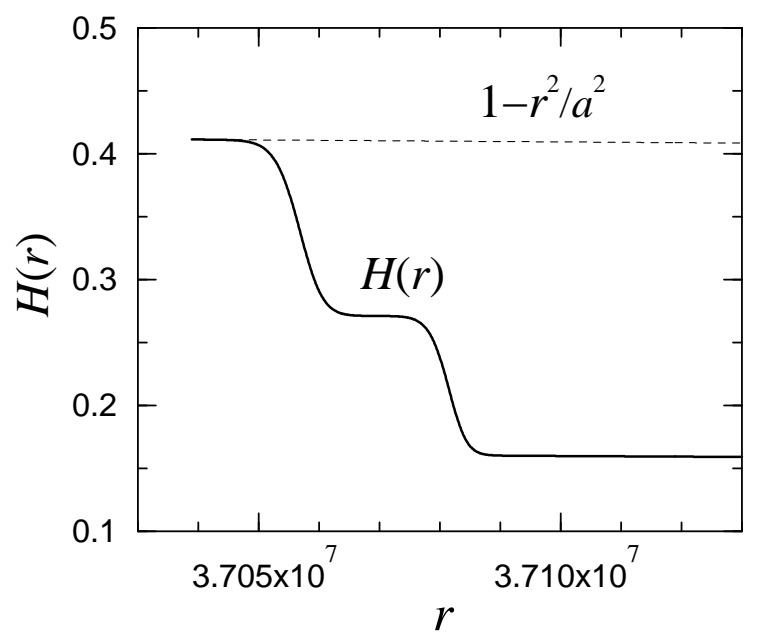

Figure 5: $H(r)$ of a solution with $f / m_{\mathrm{P}}=1.40 \times 10^{-3}, \Delta f / f=0.002, \lambda=0.01 . H(r)$ decreases in two steps in the shell region. $H(r)$ in the de Sitter space $\left(=1-r^{2} / a^{2}\right)$ is plotted in a dotted line.

extremely difficult as $f$ becomes smaller.) There are several features to be noted. First, with fixed values of $\lambda$ and $\Delta f / f$ solutions exist only for $f<f_{\max }$. For example, with $\lambda=0.01$ and $\Delta f / f=0.002$ we find that $f_{\max } \sim 0.006$. Second, as $f$ goes to $0, r_{H} / a \rightarrow 1$ and $R / a \rightarrow 0.8$. The relative size $R / a$ of the shell remains of order one even in the $f \rightarrow 0$ limit. Third, as $f$ decreases shell solutions with multiple bounces become possible. We cannot determine how many times $\phi$ can bounce inside the horizon because the numerical evaluation becomes extremely difficult when $f / m_{\mathrm{P}}<0.001$.

In the potential we are analysing, $\phi=f_{1}$ and $\phi=f_{2}$ correspond to the false and true vacua, respectively. For the existence of shell solutions the fact $V\left[f_{1}\right]>V\left[f_{2}\right]$ is not crucial,

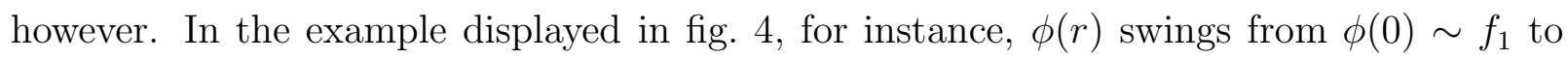
$\phi_{\max }<f_{2}$, and returns to $f_{1}$. Numerically $V\left[\phi_{\max }\right]>V[\phi(0)]$. As we are solving classical differential equations, only the form of the potential $V[\phi]$ between $f_{1}$ and $\phi_{\max }$ is relevant. The form of the potential for $\phi>\phi_{\max }$ does not matter. The second minimum can be higher than the first one; $V\left[f_{1}\right]<V\left[f_{2}\right]<V\left[\phi_{\max }\right]$.

Before concluding the section we would like to add that we have found no solution in which $\phi$ makes a transition from $f_{1}$ to $f_{2}$ as $r$ varies from 0 to $\infty$. 


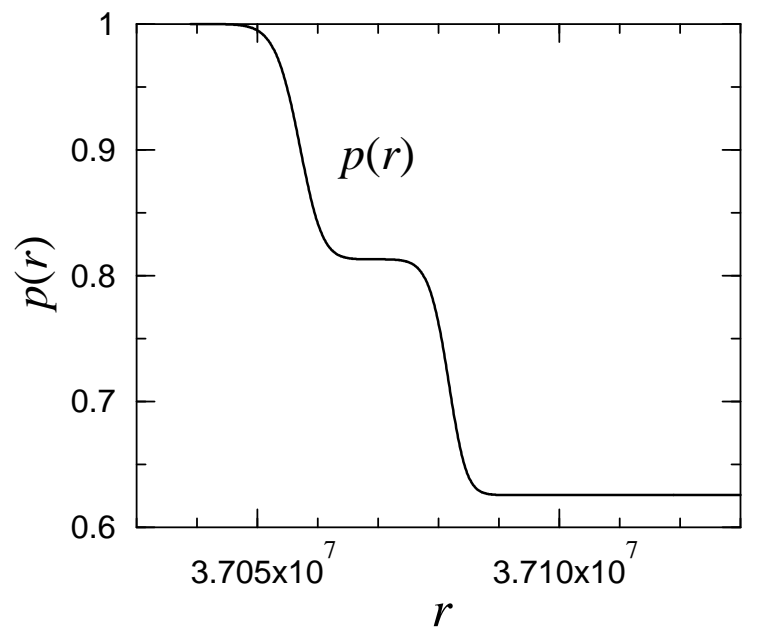

Figure 6: $p(r)$ of a solution with $f / m_{\mathrm{P}}=1.40 \times 10^{-3}, \Delta f / f=0.002, \lambda=0.01 . \quad p(r)$ decreases in two steps, from 1 to 0.6256 , in the shell region.

\section{Global Structure of the Space-Time and Solutions}

In the previous section we found novel solutions to a theory with a scalar field coupled to gravity in static coordinates. The static coordinates in (2.2), however, do not cover all of space-time. In this section we construct coordinates which allow for an extension of the solution to the full space-time manifold. These coordinates are smooth across the horizon. First we illustrate the construction with de Sitter space, and then consider the more general case which is applied to the shell solution.

The $R^{1} \times S^{3}$ metric of the de Sitter space is given by

$$
d s^{2}=a^{2}\left[-d \tau^{2}+\cosh ^{2} \tau\left(d \chi^{2}+\sin ^{2} \chi d \Omega^{2}\right)\right] .
$$

One may regard the de Sitter space as a hypersurface in the five-dimensional Minkowski spacetime constrained by the condition

$$
y_{1}^{2}+y_{2}^{2}+y_{3}^{2}+y_{4}^{2}=a^{2}+y_{0}^{2}
$$

The metric (4.1) and hypersurface (4.2) cover the entire de Sitter space, whereas the static metric covers only half of the space. Furthermore, the static metric has a coordinate singularity at $r=a$. 


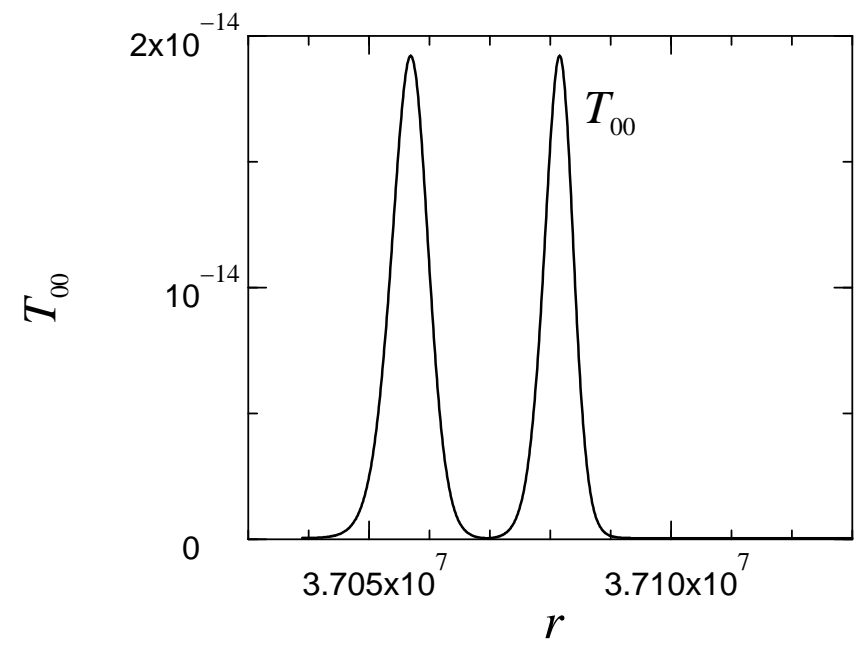

Figure 7: The energy density $T_{00}$ for a shell solution with $f / m_{\mathrm{P}}=1.40 \times 10^{-3}, \Delta f / f=$ $0.002, \lambda=0.01$. $T_{00}$ and $r$ are in the units of $m_{\mathrm{P}}^{4}$ and $l_{\mathrm{P}}$, respectively. The energy density is localized in the two shells over the de Sitter background.

The relationship among these coordinate systems are easily found. The $S^{3}$ metric (4.1) can be transformed to a metric conformal to the static Einstein universe.

$$
\begin{aligned}
& d s^{2}=\frac{a^{2}}{\cos ^{2} \eta}\left[-d \eta^{2}+d \chi^{2}+\sin ^{2} \chi d \Omega^{2}\right] \\
& \eta=\frac{\pi}{2}-2 \tan ^{-1}\left(e^{-\tau}\right), \quad-\frac{\pi}{2} \leq \eta \leq \frac{\pi}{2}
\end{aligned}
$$

Suppressing $S^{2}$, or $\theta$ and $\phi$ variables, one can map the whole de Sitter space to a square region in the $\chi-\eta$ coordinates. Null geodesics are given by straight lines at 45 degree angles.

The static and hypersurface coordinates are related by

$$
\begin{aligned}
& y_{0}=\left\{\begin{array}{rr}
\sqrt{a^{2}-r^{2}} \sinh \frac{t}{a} & \text { for } r<a \\
\sqrt{r^{2}-a^{2}} \cosh \frac{t}{a} & \text { for } r>a
\end{array}\right. \\
& y_{1}=r \cos \theta \\
& y_{2}=r \sin \theta \cos \phi \\
& y_{3}=r \sin \theta \sin \phi \\
& y_{4}=\left\{\begin{array}{rr}
\sqrt{a^{2}-r^{2}} \cosh \frac{t}{a} & \text { for } r<a \\
\sqrt{r^{2}-a^{2}} \sinh \frac{t}{a} & \text { for } r>a
\end{array}\right.
\end{aligned}
$$




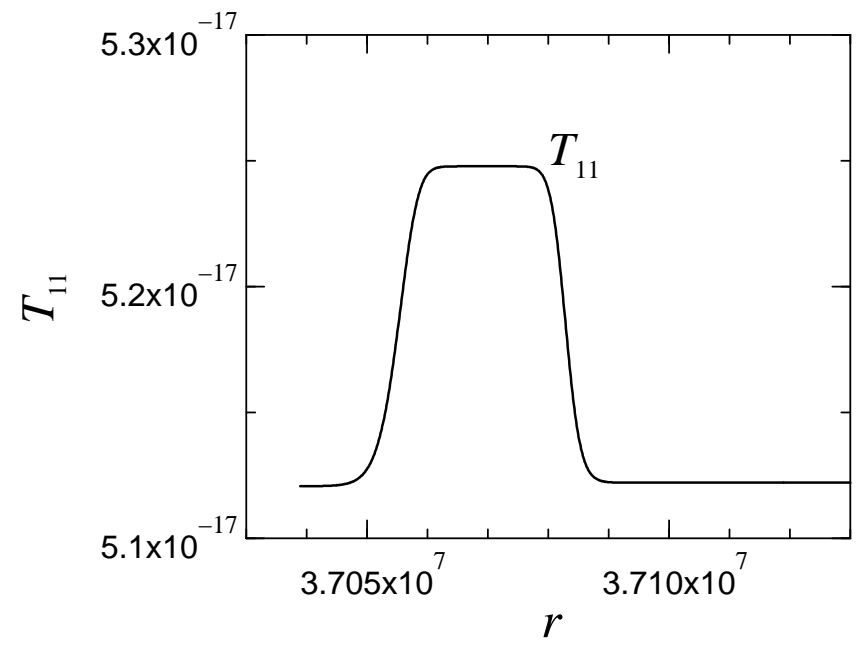

Figure 8: The radial pressure $T_{11}$ for a shell solution with $f / m_{\mathrm{P}}=1.40 \times 10^{-3}, \Delta f / f=$ $0.002, \lambda=0.01$. $T_{11}$ and $r$ are in the units of $m_{\mathrm{P}}^{4}$ and $l_{\mathrm{P}}$, respectively. The pressure is higher and constant between the two shells.

Similarly, (4.2) and (4.3) are related by

$$
\begin{aligned}
& y_{0}=a \tan \eta \\
& y_{1}=a \sec \eta \sin \chi \cos \theta \\
& y_{2}=a \sec \eta \sin \chi \sin \theta \cos \phi \\
& y_{3}=a \sec \eta \sin \chi \sin \theta \sin \phi \\
& y_{4}=a \sec \eta \cos \chi .
\end{aligned}
$$

The region inside the cosmological horizon $(r<a,-\infty<t<\infty)$ in the static metric corresponds to the left quadrant in the conformal metric $\left(0 \leq \chi \leq \frac{1}{2} \pi,|\eta|<\pi / 2-\chi\right)$ with the relations

$$
\begin{aligned}
& \frac{r}{a}=\frac{\sin \chi}{\cos \eta} \\
& \frac{t}{a}=\frac{1}{2} \ln \frac{\cos \chi+\sin \eta}{\cos \chi-\sin \eta} .
\end{aligned}
$$

Similarly, the region outside the cosmological horizon $(r>a,-\infty<t<\infty)$ in the static metric corresponds to the upper quadrant in the conformal metric $\left(0 \leq \chi \leq \pi,|\eta|>\chi-\frac{1}{2} \pi\right)$ with the relations

$$
\frac{r}{a}=\frac{\sin \chi}{\cos \eta}
$$




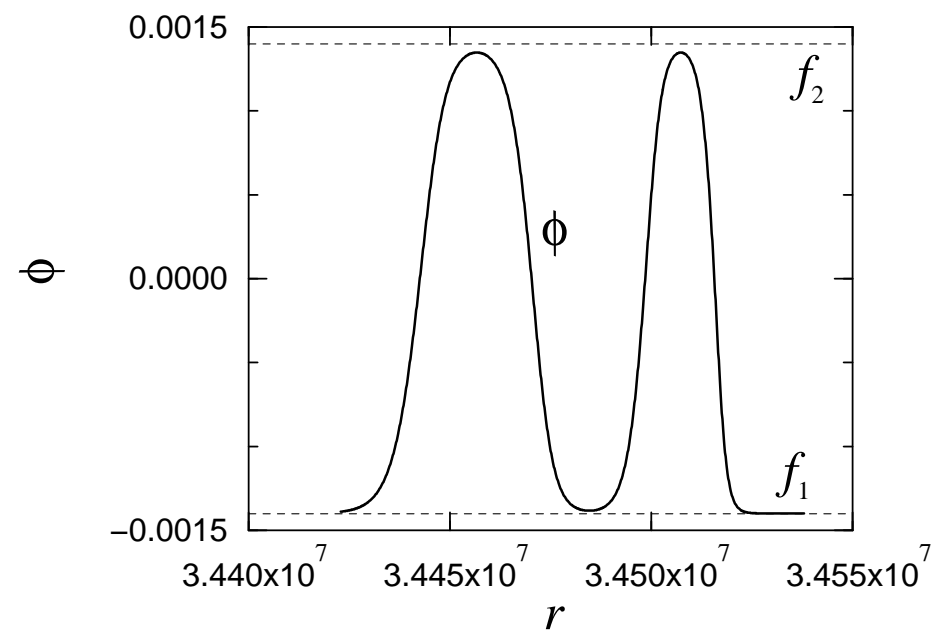

Figure 9: A solution with double bounces with $f / m_{\mathrm{P}}=1.40 \times 10^{-3}, \Delta f / f=0.002, \lambda=$ 0.01. A solution with multiple bounces appears at a smaller shell radius than a solution with a single bounce.

$$
\frac{t}{a}=\frac{1}{2} \ln \frac{\sin \eta+\cos \chi}{\sin \eta-\cos \chi} .
$$

See fig. 11 .

For the general static metric given by (2.2), with $H=H(r)$ and $p=p(r)$, the construction of the conformal coordinates analogous to (4.3) proceeds as follows. We suppose that $H(r)$ has a single zero at $r_{H}$ whereas $p(r)>0$. The new radial coordinate is defined by

$$
r_{*}(r)= \begin{cases}\int_{0}^{r} d r^{\prime} \frac{p\left(r^{\prime}\right)}{H\left(r^{\prime}\right)} & \text { for } r<r_{H} \\ \int_{\infty}^{r} d r^{\prime} \frac{p\left(r^{\prime}\right)}{H\left(r^{\prime}\right)} & \text { for } r>r_{H} .\end{cases}
$$

It has a logarithmic singularity at $r_{H}$, diverging there as $r_{*} \sim-\frac{1}{2} b \ln \left|r-r_{H}\right|$ where $b=$ $-2 p\left(r_{H}\right) / H^{\prime}\left(r_{H}\right)>0$. New coordinates are introduced by

$$
\begin{aligned}
\tan u & =+e^{+t / b} e^{-r_{*} / b} \\
\tan v & =\mp e^{-t / b} e^{-r_{*} / b} .
\end{aligned}
$$

The upper sign is for $r<r_{H}$ and the lower sign is for $r>r_{H}$. The $u-v$ coordinates are related to the $U-V$ coordinates in the Kruskal-Szekeres (KS) coordinate system [16] for 


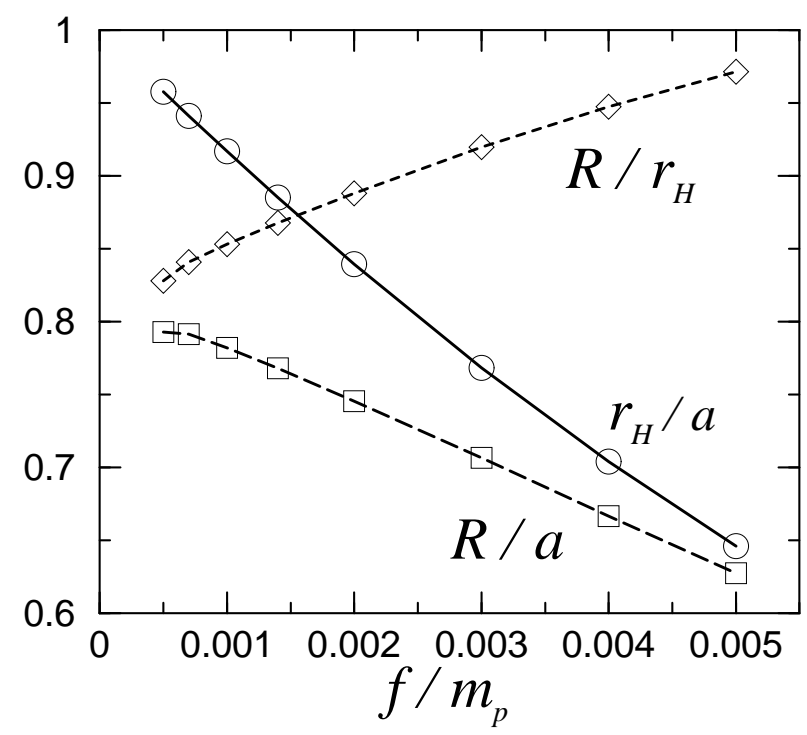

Figure 10: The $f$-dependence of the shell solutions. $\lambda=0.01$ and $\Delta f / f=0.002$ are fixed. $R, r_{H}$, and $a$ are the radius of the shell, the horizon length where $H(r)$ vanishes, and the horizon length in the de Sitter space, respectively. The ratios of various pairs are plotted. Circles, squares, and diamonds correspond to data points obtained.

the Schwarschild solution, and to the Gibbons-Hawking (GH) coordinate system [17 for de Sitter space. The connection between the KS and GH coordinate systems has already been discussed in [12]. Essentially $\tan u=U+V$ and $\tan v=V-U$. The metric becomes

$$
\begin{aligned}
d s^{2} & =-\frac{4 b^{2} F(u, v)}{\cos ^{2}(u+v)} d u d v+r^{2} d \Omega^{2} \\
F(u, v) & =\frac{H}{4 p^{2}}(1-\tan u \tan v)(1-\cot u \cot v) \\
& =\frac{H}{2 p^{2}}\left(1 \pm \cosh \frac{2 r_{*}}{b}\right) \quad \text { for }\left\{\begin{array}{l}
r \leq r_{H} \\
r \geq r_{H}
\end{array} .\right.
\end{aligned}
$$

The static metric covers the region interior to the bounding lines $u=0, u+v=\frac{1}{2} \pi$, and $u-v=\frac{1}{2} \pi$. The horizon in the static metric, $r=r_{H}$, corresponds to the single point $u=v=0$. The function $F$ is non-vanishing and finite there as $e^{2 r_{*} / b} \sim 1 /\left|r-r_{H}\right|$. In the $u-v$ coordinates the metric is regular on $u=0$ so that the extension to the region $u<0$ can be made, whereas the static metric covers only half of the space.

Applied to the de Sitter space we find

$$
r_{H}=b=a, \quad e^{-r_{*} / b}=\left|\frac{a-r}{a+r}\right|^{1 / 2}, \quad F=1,
$$




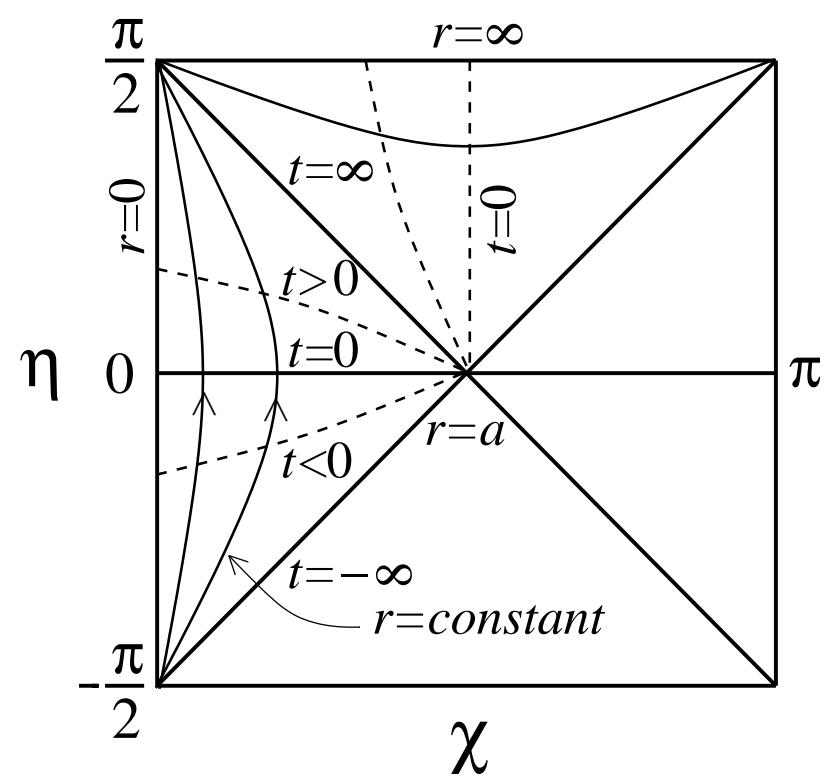

Figure 11: Penrose diagram of the de Sitter space.

and the metric (4.3) is recovered by

$$
\eta=u+v, \quad \chi=v-u+\frac{\pi}{2}
$$

The shell solution found in the preceeding sections can be extended to the entire spacetime. In the region III $\left(R_{2}<r\right)$ defined in the static metric $H(r)$ is given by $(2.23)$, and the location of the horizon is determined by $H\left(r_{H}\right)=0$. In region III, $\delta \phi(r)$ is very small so that its equation of motion can be linearized. We divide region III into two; region IIIa $\left(R_{2}<r<2 r_{H}-R_{2}\right)$ and region IIIb $\left(r>2 r_{H}-R_{2}\right)$. In region IIIa, $H(r)$ can be approximated by

$$
H(r) \sim A\left(1-\frac{r^{2}}{r_{H}^{2}}\right)
$$

where

$$
A=\frac{1}{2}\left(\frac{3 r_{H}^{2}}{a^{2}}-1\right)
$$

The coefficient $A$ has been chosen such that both (2.23) and (4.13) have the same slope $H^{\prime}\left(r_{H}\right)$ at the horizon. In the examples described in Section 3, errors caused by (4.13) are less than $15 \%$ in region IIIa. Now we write the linearized version of (2.11) in terms of 
$y=1-\left(r^{2} / r_{H}^{2}\right)$.

$$
\begin{aligned}
& \left\{y(1-y) \frac{d^{2}}{d y^{2}}+\left(1-\frac{5}{2} y\right) \frac{d}{d y}-\left(\tilde{\kappa}^{2}+\frac{9}{16}\right)\right\} \delta \phi=0 \\
& A\left(\tilde{\kappa}^{2}+\frac{9}{16}\right)=\kappa^{2}+\frac{9}{16}=\frac{1}{4} \omega^{2} a^{2}
\end{aligned}
$$

Since $\delta \phi(r)$ must be regular at $r=r_{H}(y=0)$, the solution is

$$
\delta \phi(r)=\delta \phi\left(r_{H}\right) \cdot F\left(\frac{3}{4}+i \tilde{\kappa}, \frac{3}{4}-i \tilde{\kappa}, 1 ; y\right)
$$

The normalization $\delta \phi\left(r_{H}\right)$ must be such that $\delta \phi(r)$ matches at $r=R_{2}$ with the value determined by numerical integration in region II. Essentially $\delta \phi(r)$ decreases exponentially when $R_{2}<r<r_{H}$.

Near $r=R_{2}$ the hypergeometric function behaves as

$$
F\left(\frac{3}{4}+i \tilde{\kappa}, \frac{3}{4}-i \tilde{\kappa}, 1 ; y\right) \sim \frac{1}{2 \sqrt{\pi \tilde{\kappa}}} y^{-1 / 4}(1-y)^{-1 / 2} \exp \left\{2 \widetilde{\kappa} \sin ^{-1} \sqrt{y}\right\}
$$

so that

$$
\frac{\delta \phi^{\prime}(r)}{\delta \phi(r)}=-\frac{2 r}{r_{H}^{2}} \cdot \frac{\tilde{\kappa}}{\sqrt{y(1-y)}} .
$$

The value of the right side of (4.18) at $r=R_{2}$ can be compared with the value obtained by direct numerical integration in region II. In one example with $f / m_{\mathrm{P}}=2 \times 10^{-3}, \Delta f / f=$ $2 \times 10^{-3}, \lambda=0.01, R_{2}=1.765 \times 10^{7}, a=2.365 \times 10^{7}, r_{H}=1.985 \times 10^{7}, \kappa=3344$, and $\tilde{\kappa}=4481$. The numerical value for $\delta \phi^{\prime}\left(R_{2}\right) / \delta \phi\left(R_{2}\right)$ is -0.000848 , whereas the value from (4.18) is -0.000985 . With the uncertainty in the value of $\tilde{\kappa}$ caused by the approximation (4.13) taken into account, one may conclude that the agreement is rather good. To gauge the difficulty of determining the solution numerically for all values of $r$, we note that $\delta \phi\left(r_{H}\right) \sim 10^{-1850} \cdot \delta \phi\left(R_{2}\right)$.

The most important observation is that the solution $\phi(r)$ is regular at the horizon, $r=r_{H}$, though the static metric $(t, r, \theta, \phi)$ is not: $r=r_{H}$ in the static metric is a coordinate singularity. As seen above, $r=r_{H}$ corresponds to $u=v=0$ in the conformal metric (4.10) which is non-singular. Near the horizon $\tan u \tan v \sim r-r_{H}$ so that

$$
\phi=f_{1}+\delta \phi\left(r_{H}\right)\left\{1-\frac{2}{r_{H}}\left(\tilde{\kappa}^{2}+\frac{9}{16}\right) u v+\cdots\right\} .
$$


Furthermore, as $\tan u \tan v=e^{-2 r_{*}(r) / b}$, the static solution $\phi(u, v)$ depends only on $\tan u \tan v$ in the entire space-time. This implies that

$$
\phi(-u,-v)=\phi(u, v) .
$$

In the $\eta$ - $\chi$ coordinates $\left(S^{3}\right.$-metric) defined by (4.12)

$$
\phi(\eta, \chi)=\phi(-\eta, \pi-\chi)=\phi(\eta, \pi-\chi) .
$$

This relation shows that there is a mirror image of the shell structure in the other hemisphere in the $S^{3}$-metric. See fig. 12. The shaded regions in the figure represent the shells. The size of the shells is invariant, as is obvious in the static coordinates. In the $S^{3}$ or conformal metric an observer at the center $(\chi=0$ or $\pi)$ sends a light signal toward the shell. Another observer at the shell, upon receiving the signal from the center, sent a signal back to the observer at the center. The total elapsed time, in terms of the proper time of the observer at the center $a \tau$ where $\tau$ is related to $\eta$ by (4.3) at $\chi=0$, is $a \ln (a+R) /(a-R)$, independent of when the signal is emitted initially, as straightforward manipulations show.

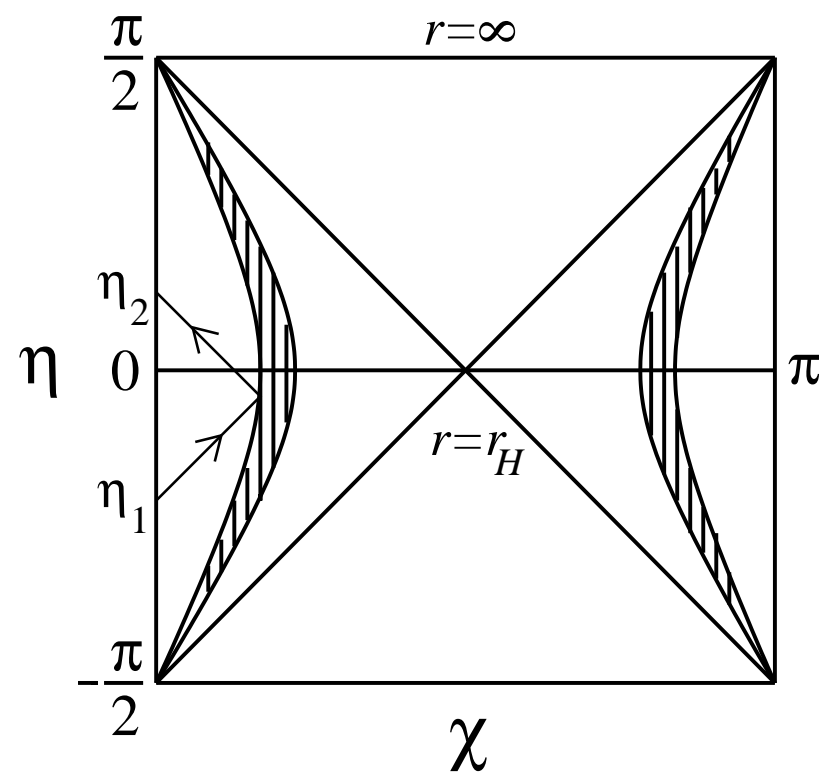

Figure 12: Global structure of the shell solution in the conformal coordinates. The mirror image appears in the other hemisphere. 
Finally, in the region outside the horizon in the static metric, or in the upper or lower quadrant in the Penrose diagram, $\delta \phi(r)$ decays as $r^{-3 / 2}$. The proof goes as follows. For $r \gg r_{H}, H \sim 1-\left(r^{2} / a^{2}\right)$, so that $\delta \phi(r)$ satisfies Eq. (2.17). Hence it is a linear combination

of $u_{1}=F\left(\frac{3}{4}+i \kappa, \frac{3}{4}-i \kappa, \frac{3}{2} ; z\right)$ and $u_{2}=z^{-1 / 2} F\left(\frac{1}{4}+i \kappa, \frac{1}{4}-i \kappa, \frac{1}{2} ; z\right)$. These can be written as

$$
\begin{aligned}
{\left[\begin{array}{l}
u_{1} \\
u_{2}
\end{array}\right] } & =\left[\begin{array}{c}
\frac{1}{2} \Gamma\left(\frac{3}{4}-i \kappa\right)^{-2} \\
\Gamma\left(\frac{1}{4}-i \kappa\right)^{-2}
\end{array}\right] \frac{\sqrt{\pi} \Gamma(-2 i \kappa)}{(-z)^{(3 / 4)+i \kappa}} F\left(\frac{3}{4}+i \kappa, \frac{1}{4}+i \kappa, 1+2 i \kappa ; \frac{1}{z}\right) \\
& +\left[\begin{array}{c}
\frac{1}{2} \Gamma\left(\frac{3}{4}+i \kappa\right)^{-2} \\
\Gamma\left(\frac{1}{4}+i \kappa\right)^{-2}
\end{array}\right] \frac{\sqrt{\pi} \Gamma(+2 i \kappa)}{(-z)^{(3 / 4)-i \kappa}} F\left(\frac{3}{4}-i \kappa, \frac{1}{4}-i \kappa, 1-2 i \kappa ; \frac{1}{z}\right) .
\end{aligned}
$$

From this one can write

$$
\phi \sim f_{1}+A\left(\frac{a}{r}\right)^{3 / 2} \sin \left(2 \kappa \ln \frac{r}{a}+\delta\right)
$$

when $r \gg r_{H}$.

\section{False Vacuum Decay}

If the region inside the shell is in a false vacuum state one should consider its quantum decay to the true vacuum state. The lifetime of the false vacuum may be determined semi-classically using the methods of Coleman et al. without [18] or with [6] gravity taken into account. The rate per unit volume for making a transition from the false vacuum to the true vacuum is expressed as

$$
\frac{\Gamma}{V}=A e^{-B / \hbar}[1+\mathcal{O}(\hbar)]
$$

where Planck's constant has been used here to emphasize the semi-classical nature of the tunneling rate. For the potential being used in this paper we find the $\mathrm{O}(4)$, Euclidean space, bounce action (neglecting gravity) to be

$$
B_{0}=\frac{36 \pi^{2}}{\lambda}\left(\frac{f}{\Delta f}\right)^{3}
$$

where the radius of the critical size bubble which nucleates the transition is

$$
\rho_{c}=\frac{3}{\Delta f} \sqrt{\frac{2}{\lambda}} .
$$


For any sensible estimate of the coefficient $A$ the lifetime of the false vacuum will exceed the present age of the universe when the condition

$$
\lambda\left(\frac{\Delta f}{f}\right)^{3}<1
$$

is satisfied. This calculation is based on the thin wall approximation, which is valid when the critical radius is large compared to the coherence length of the potential, namely $1 / \sqrt{\left|V^{\prime \prime}\right|}$. This condition translates into

$$
\Delta f \ll 6 f .
$$

With gravity included the bounce action is

$$
B=\frac{B_{0}}{\left[1+\left(\rho_{c} / 2 R\right)^{2}\right]^{2}} .
$$

Gravitational effects are negligible when $\rho_{c}<R$. When this condition is not fulfilled the shell radius is too small to accommodate even a single nucleation bubble and therefore nucleation is further suppressed.

\section{Summary}

In this paper we have reported the discovery of shell-like solutions to the combined field equations of gravity and a scalar field with a double-minima potential. These solutions exist in a space that is asymptotically de Sitter. The range of parameters which allow such solutions are very broad. If anything like these structures exist in nature they most likely would have been created in the early universe and are therefore cosmological. We know of no other way to produce them.

To make matters interesting, let us suppose that the cosmological constant suggested by recent observations of distant Type Ia supernovae [19] arises from the universe actually being in a false vacuum state. A best fit to all cosmological data 20 reveals that the present energy density of the universe has the critical value of $\epsilon_{c}=3 H_{0}^{2} / 8 \pi G$, with one-third of it consisting of ordinary matter and two-thirds of it contributed by the cosmological constant. Suppose that the cosmological constant arises from a potential of the form we have been 
analyzing. Then with a present value of the Hubble constant of $H_{0}=65 \mathrm{~km} / \mathrm{s} \cdot \mathrm{Mpc}$ we find that

$$
a=\sqrt{\frac{3}{2}} \frac{1}{H_{0}}=1.7 \times 10^{26} \mathrm{~m}
$$

and so

$$
\left(\lambda f^{3} \Delta f\right)^{1 / 4}=2.4 \times 10^{-3} \mathrm{eV}
$$

This is a constraint on the parameters of the potential, $\lambda, f$ and $\Delta f$. Although they cannot be determined individually from this data, we can place limits on them such that a shell structure might arise. Recalling (2.26) we find

$$
\begin{aligned}
& f<30 \lambda^{-1 / 6} \mathrm{MeV} \\
& w>6.3 \lambda^{-1 / 3} \mathrm{fm} .
\end{aligned}
$$

This is pure speculation of course. A cosmological constant, if it exists, may have its origins elsewhere. But if it does arise from a false vacuum, a variety of questions immediately present themselves. Is $\phi$ a new field, not present in the standard model of particle physics, whose only purpose is this? Where does the energy scale of $2.4 \mathrm{meV}$ come from? Why should $V[\phi]$ have a global minimum of 0 , especially when quantum mechanical fluctuations are taken into account? To these questions we have no answers.

\section{Acknowledgments}

This work was supported by the US Department of Energy under Grant DE-FG0287ER40328, and by the Japan Ministry of Education and Science under Grants No. 13640284 and No. 13135215.

\section{References}

[1] J. Bjoraker and Y. Hosotani, Phys. Rev. Lett. 84, 1853 (2000);

J. Bjoraker and Y. Hosotani, Phys. Rev. D 62, 43513 (2000);

Y. Hosotani, gr-qc/0103069, to appear in J. Math. Phys.

[2] E. Radu, gr-qc/0109015.

[3] D. V. Gal'tsov, hep-th/0112038.

[4] S. Deser, Phys. Rev. D 64, 463 (1976). 
[5] R. Bartnik and J. McKinnon, Phys. Rev. Lett. 61, 141 (1988);

H. Künzle and A. Masood-ul-Alam, J. Math. Phys. 31, 928 (1990);

J. A. Smoller, A. G. Wasserman, S. T. Yau and J. B. McLeod, Comm. Math. Phys. 143, 115 (1991);

J. A. Smoller and A. G. Wasserman,, ibid. 151, 303 (1993);

M. S. Volkov, N. Straumann, G. Lavrelashvili, M. Huesler and O. Brodbeck, Phys. Rev. D 54, 7243 (1996).

[6] S. Coleman and F. De Luccia, Phys. Rev. D 21, 3305 (1980).

[7] R. G. Daghigh, J. I. Kapusta, and Y. Hosotani, gr-qc/0008006 (unpublished);

[8] D. V. Gal'tsov and J. P. S. Lemos, Class. Quant. Grav. 18, 1715 (2001).

[9] K. A. Bronnikov, Phys. Rev. D64, 064013 (2001);

K. A. Bronnikov and G. N. Shikin, gr-qc/0109027.

[10] K. Maeda, K. Sato, M. Sasaki and H. Kodama, Phys. Lett. B108, 98 (1982);

K. Sato, H. Kodama, M. Sasaki and K. Maeda, ibid. 103 (1982).

[11] K. Sato, M. Sasaki, H. Kodama, and K. Maeda, Prog. Theoret. Phys. 65, 1443 (1981);

H. Kodama, M. Sasaki, K. Sato, and K. Maeda, ibid. 66, 2052 (1981);

H. Kodama, M. Sasaki, and K. Sato, ibid. 68, 1979 (1982);

K. Sato, ibid. 66, 2287 (1981).

[12] S. K. Blau, E. I. Guendelman and A. H. Guth, Phys. Rev. D 35, 1747 (1987);

E. Fahri, A. H. Guth, and J. Guven, Nucl. Phys. B339, 417 (1990).

[13] A preliminary result has been reported in

Y. Hosotani, Soryushiron Kenkyu 103, E91 (2001); hep-th/0104006.

[14] I. G. Dymnikova, Phys. Lett. B472, 33 (2000); gr-qc/0010016.

[15] Bateman Manuscript Project, vol. 1. §2.3.2, page 77.

[16] M. D. Kruskal, Phys. Rev. 119, 1743 (1960);

G. Szekeres, Publ. Mat. Debrecen 7, 285 (1960).

[17] G. W. Gibbons and S. W. Hawking, Phys. Rev. D 15, 2738 (1977).

[18] S. Coleman, Phys. Rev. D 15, 2929 (1977); 16, 1248(E) (1977);

C. G. Callan and S. Coleman, ibid. 16, 1762 (1977).

[19] A. G. Riess et al., Astron. J. 116, 1009 (1998);

P. M. Garnavich et al., Astrophys. J. 509, 74 (1998);

S. Perlmutter et al., Astrophys. J. 517, 565 (1999).

[20] N. A. Bahcall, J. P. Ostriker, S. Perlmutter and P. J. Steinhardt, Science 284, 1481 (1999). 